\title{
Forward energy and jet flows in high energy factorisation
}

\section{Michal Deák*i}

Instituto de Física Teórica UAM/CSIC, Facultad de Ciencias, C-XVI,

Universidad Autónoma de Madrid, Cantoblanco, Madrid 28049, SPAIN

E-mail: michal.deak@desy.de

The observables relevant for forward jets at LHC are calculated. The simulations are performed using Monte Carlo event generators. In particular we compare results from CASCADE based on high energy factorization and PYTHIA which is based on collinear factorization.

XVIII International Workshop on Deep-Inelastic Scattering and Related Subjects, DIS 2010

April 19-23, 2010

Firenze, Italy

\footnotetext{
*Speaker.

${ }^{\dagger}$ I would like to thank organizers for a very nice meeting. The results presented in this article have been obtained in collaboration with K. Kutak, H. Jung, F. Hautmann.
} 


\section{Introduction}

Experiments at the Large Hadron Collider (LHC) will allow to test the Standard Model at very high energies. From the point of view of Quantum Chromodynamics (QCD) at the LHC it will be possible to probe the partonic structure of the proton at extreme energies. Jet production and forward jet production in particular $[1,2]$ is of big interest because it probes dynamics of partons carrying very small fractions of the proton momentum. By having a large center of mass energy at the LHC $\sqrt{s}$ and hard subprocess at a lower energy scale $M$, so $\Lambda_{Q C D}^{2} \ll M^{2} \ll s$, will require application of QCD resummation approaches capable to account for multiple scales [3, 4]. Namely, one has to account for logarithms of type $\alpha_{s}^{n} \ln ^{m} p_{\perp} / \Lambda_{Q C D}$ where $p_{\perp}$ is a transversal momentum of produced jet and another type of logarithms: $\alpha_{s}^{n} \ln ^{m} 1 / x[5,6]$ due to the fact that one of the incoming protons will be probed at very small longitudinal momentum fraction. The theoretical framework to resum consistently both kinds of logarithmic corrections in pQCD is based on high-energy factorization at fixed transverse momentum [7, 8, 9]. This formulation depends on unintegrated parton distributions, obeying appropriate evolution equations, and short-distance, process-dependent matrix elements. The unintegrated-level evolution is given by evolution equations in rapidity, or angle, parameters. Different forms of the evolution, valid in different kinematic regions, are available, see $[10,11,12,13]$, and references therein. In this article we apply recently obtained [14] results for hard matrix elements relevant for forward jet physics together with parton shower Monte Carlo generator CASCADE [15] for calculating observables related to forward jet phenomenon. The paper is organized as follows. In Sec. 2 we recall elements of high energy factorization framework relevant for our study. In Sec. 3 we present phenomenological results for jet production focusing on transversal momentum spectra and rapidity spectra.

\section{Factorization kinematics and matrix elements relevant for forward jets}

Let us now consider a final state in which a hard forward jet, a jet with high rapidity, and a hard central jet are tagged. Each of the jets can be associated with a group of high $p_{\perp}$ partons. The high $p_{\perp}$ production at microscopic level can be understood as originating from collision of two partons where one of them which is almost on-shell carries large longitudinal momentum fraction $\xi_{1} p_{1}$ of mother proton $\left(p_{1}\right)$ while the other one carries small longitudinal momentum fraction $\xi_{2} p_{2}$ of the other proton $\left(p_{2}\right)$ and is off-shell, where $k_{1}, k_{2}$, are the four momenta of initial state partons and $p_{3}$ and $p_{4}$ are four-momenta of final state partons.

The framework to describe forward jets is provided by high-energy factorization which was derived after observation of gluon exchange dominance at high energies. Similarly to collinear factorization it decomposes cross-section into parton density functions characterizing incoming hadrons $\phi\left(\xi, k_{\perp}\right)$ at fixed transverse momentum, and perturbatively calculable matrix elements $\widehat{\sigma}$. However, it resums apart from large logarithms of hard scale also large logarithms coming from energy ordering. The formula for high energy factorization while applied to considered here process assumes form:

$$
\sigma=\sum_{a} \int d \xi_{1} d \xi_{2} d^{2} k_{T} \phi_{a / A}\left(\xi_{1}, \mu^{2}\right) \widehat{\sigma}\left(\xi_{1} \xi_{2} S, Q_{T}, k_{T}, \varphi\right) \phi_{g^{*} / B}\left(\xi_{2}, k_{T}, \mu^{2}\right)
$$



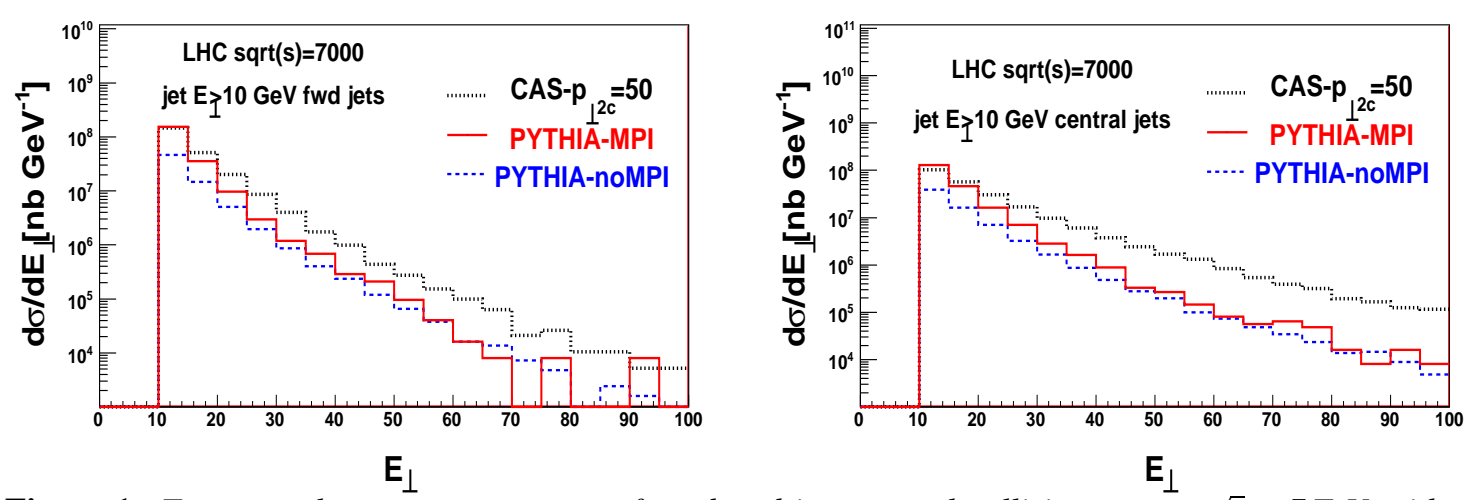

Figure 1: Transversal momentum spectra of produced jets at total collision energy $\sqrt{s}=7$ TeV with requirement that $p_{\perp}>10 \mathrm{GeV}$. We compare predictions obtained from CASCADE and PYTHIA running in a multiple interactions mode and no multiple interactions mode. Spectrum of forward jets (left); spectrum of central jets (right).

where as example we took total cross section and where sum runs over quark flavors. In highenergy factorization framework the parton densities are solutions to integro-differential evolution equations summing up perturbative terms with strong ordering condition in rapidity or angle of subsequently emitted partons. Such equations should be supplemented with some nonperturbative input distribution at initial value of ordering parameter which then is evolved with the evolution equation towards larger value of ordering parameter. The matrix elements relevant for high energy factorization describe hard subprocess where at least one of incoming partons is off mass shell. They are calculated by applying to scattering amplitudes $\mathscr{M}$ the high-energy eikonal projectors. In reference [14] matrix elements relevant for forward jets phenomenology have been calculated, in fully exclusive form.

\section{Forward jet phenomenology at the LHC}

We calculate forward jet cross sections for a typical experimental scenario at LHC. We require at least two jets with $\mathrm{E}_{\perp}>10 \mathrm{GeV}$, where one jet has to be detected in the central region defined by $\left|\eta_{c}\right|<2$ and the other jet is reconstructed in the forward region defined by $3<|\eta|<5$. The jets are reconstructed using the invariant $a n t i-k_{t}$-algorithm. We compare predictions from running the CASCADE Monte Carlo event generator with the PYTHIA [16] Monte Carlo event generator running in two modes: with and without multi-parton interactions. Both Monte Carlo generators simulate higher order QCD corrections with parton showers: CASCADE uses parton showers according to the CCFM evolution equation whereas PYTHIA uses DGLAP based parton showers.

\subsection{Transverse momentum spectra}

In Fig. 1 the prediction of differential cross section $\frac{d \sigma}{d p_{\perp}}$ is shown as obtained from CASCADE 

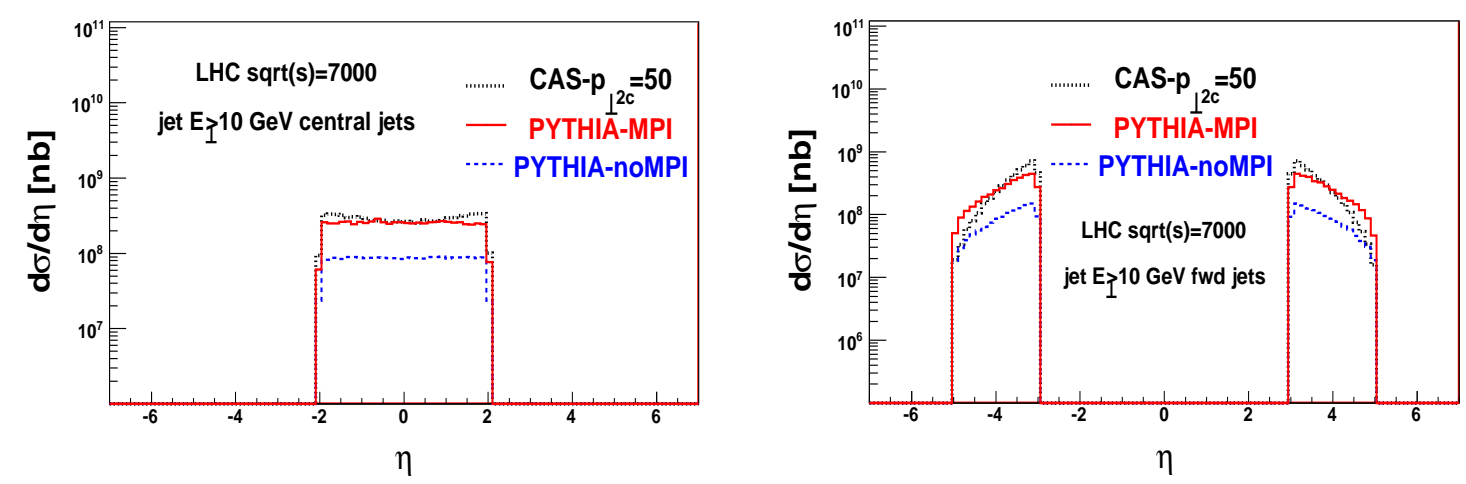

Figure 2: Pseudorapidity spectra of produced jets at total collision energy $\sqrt{s}=7 \mathrm{TeV}$ with requirement that $p_{T}>10 \mathrm{GeV}$. We compare predictions obtained from CASCADE and PYTHIA running in multiple interactions mode and no multiple interactions mode. Spectrum of forward jets (left); spectrum of central jets (right).

and PythiA. The cross sections predicted from both simulations at low momentum are of the similar order, however, at larger transverse momentum the CASCADE predicts a larger cross section what is clearly visible for central jets (Fig. 1 right). This behavior is expected since CASCADE uses matrix elements which are calculated within high energy factorization scheme allowing for harder transversal momentum dependence as compared to collinear factorization. Moreover CASCADE applies CCFM parton shower utilizing angle dependent evolution kernel which at small $x$ does not lead to ordering in transverse momentum, and thus allow for more hard radiations during evolution as compared to based on leading order DGLAP splitting functions Monte Carlo generator PYTHIA. The parton shower has major influence on the side where the small $x$ gluon enters the hard interaction, thus the jets in the central region are mainly affected by the parton shower.

\subsection{Rapidity dependence}

In fig. 2 we show prediction for pseudorapidity dependence of the cross section in two regions $0<|\eta|<2$ and $3<|\eta|<5$. We see that results from CASCADE interpolate between PyTHIA with multiple interactions in the central region and PYTHIA without multiple interactions in the forward region. The result is due to the fact that CASCADE (because of angular ordering), and PYTHIA with multiple interactions (because of multi chain exchanges), predict more hadronic activity in the central rapidity region as compared to the collinear shower. In the remaining rapidity region cascade uses collinear parton shower of a similar type as in PYTHIA without multiple interactions.

\section{References}

[1] X. Aslanoglou et al., "Performance Studies of Prototype II for the CASTOR forward Calorimeter at Eur. Phys. J. C 52, 495 (2007). 
[2] Z. J. Ajaltouni et al., "Proceedings of the workshop: HERA and the LHC workshop series on the arXiv:0903.3861 [hep-ph].

[3] W. J. Stirling, "Production Of Jet Pairs At Large Relative Rapidity In Hadron Hadron Nucl. Phys. B 423, 56 (1994).

[4] V. Del Duca, M. E. Peskin and W. K. Tang, Phys. Lett. B 306, 151 (1993).

[5] L. N. Lipatov, "Reggeization Of The Vector Meson And The Vacuum Singularity In Nonabelian Sov. J. Nucl. Phys. 23, 338 (1976) [Yad. Fiz. 23, 642 (1976)].

[6] E. A. Kuraev, L. N. Lipatov and V. S. Fadin, Sov. Phys. JETP 45, 199 (1977) [Zh. Eksp. Teor. Fiz. 72, 377 (1977)].

[7] S. Catani, M. Ciafaloni and F. Hautmann, Phys. Lett. B 242, 97 (1990).

[8] S. Catani, M. Ciafaloni and F. Hautmann, Nucl. Phys. B 366, 135 (1991).

[9] S. Catani, M. Ciafaloni and F. Hautmann, Phys. Lett. B 307, 147 (1993).

[10] J. Collins, PoS LC2008, 028 (2008).

[11] F. Hautmann, Acta Phys. Polon. B 40, 2139 (2009).

[12] T. C. Rogers, "Next-to-Leading Order Hard Scattering Using Fully Unintegrated Parton Phys. Rev. D 78, 074018 (2008) [arXiv:0807.2430 [hep-ph]].

[13] F. Hautmann, Phys. Lett. B 655, 26 (2007) [arXiv:hep-ph/0702196].

[14] M. Deak, F. Hautmann, H. Jung and K. Kutak, JHEP 0909 (2009) 121.

[15] H. Jung, Comput. Phys. Commun. 143, 100 (2002).

[16] T. Sjostrand, S. Mrenna and P. Z. Skands, JHEP 0605 (2006) 026 